\title{
Non-relativistic Extended Gravity and its applications across different astrophysical scales
}

\author{
J. C. Hidalgo, S. Mendoza, X. Hernandez, T. Bernal, M. A. Jimenez and \\ C. Allen \\ Instituto de Astronomía, Universidad Nacional Autónoma de México, AP 70-264, Distrito Federal \\ 04510, México.
}

\begin{abstract}
Using dimensional analysis techniques we present an extension of Newton's gravitational theory built under the assumption that Milgrom's acceleration constant is a fundamental quantity of nature. The gravitational force converges to Newton's gravity and to a MOND-like description in two different mass and length regimes. It is shown that a modification on the force sector (and not in the dynamical one as MOND does) is more convenient and can reproduce and predict different phenomena usually ascribed to dark matter at the non-relativistic level.
\end{abstract}

Keywords: Gravitation, binaries:general, galaxies: star clusters: general, galaxies: kinematics and dynamics.

PACS: $04.50 . \mathrm{Kd}, 98.62 . \mathrm{-g}, 98.62 . \mathrm{Dm}, 98.10 .+\mathrm{z}$

\section{EXTENDED GRAVITY}

The gravitational anomalies in the dynamics of galaxies and larger systems are the basis to expect non-baryonic dark matter. Since gravitational experiments have been performed in systems no larger than the mass and length scales associated to those of the Solar System, then it is necessary to explore an extension of the gravitational theory avoiding an elusive dark matter component.

Milgrom [1] developed a modification in the dynamical sector of Newton's second law in such a way that, when a test particle experiences a gravitational acceleration greater than an acceleration constant $a_{0} \sim 10^{-10} \mathrm{~m} / \mathrm{s}^{2}$, a Newtonian behaviour is expected and, when the inequality is inverted, the acceleration exerted is proportional to the square root of the Newtonian value. A relativistic Tensor-Vector-Scalar (TeVeS [2]) generalisation of this has been proposed, but its inconsistency with gravitational lensing [3] and the null-observations by the terrestrial Newtonian law of inertia experiments indicate that the modification has to be done in the gravitational sector and not in the dynamical one.

Here we assume that Milgrom's acceleration constant $a_{0}$ is of fundamental character. The acceleration $a$ felt by a test particle at a distance $r$ from the mass $M$ generating the gravitational field must also be a function of Newton's constant of gravity $G$, therefore, 
Buckingham's theorem of dimensional analysis demands that [4]:

$$
a=a_{0} f(x) \rightarrow a_{0}\left\{\begin{array}{l}
x:=l_{M} / r:=\left(G M a_{0}^{-1}\right)^{1 / 2} / r, \quad \text { for } x \ll 1, \\
x^{2}=\left(l_{M} / r\right)^{2}=G M a_{0}^{-1} / r^{2}, \quad \text { for } x \gg 1 .
\end{array}\right.
$$

As explained by [4], where the theory first appeared, the introduction of a mass-length scale $l_{M}$ breaks the scale-invariance of gravity. This Extended Newtonian theory of gravity (ENG) modifies the gravitational force and leaves the inertia law intact. The force law is constructed from expansions in Taylor series about the limits (1), obtaining [4]:

$$
a / a_{0}=f(x)=x\left(1-x^{n+1}\right) /\left(1-x^{n}\right),
$$

where the parameter $n$ modulates the smoothness of the transition between the Newtonian and the MONDian regimes. In fact, a fit to the rotation profile of the Milky Way indicates that $n=3$ [4]. Also, Newton's theorems remain valid on spherically symmetric matter distributions.

\section{TESTS AND PROBES ACROSS ASTROPHYSICAL SCALES}

A few testable and falsifiable predictions can be derived in the ENG since the experimental confirmation of Newtonian gravity for $x<1$ would discard the theory completely. Conversely, the theory would not be an alternative if observations infer the existence of dark matter at scales above $x=1$. In regard of this, we have explored environments to probe gravity at the non-relativistic level.

In [4], the predictions of ENG were tested over several astrophysical scales. In our Solar System, departures from the Newtonian acceleration (2) lie within the uncertainty of the orbits of planets. Recently [5] has shown the same trend for the measured acceleration of the Moon's orbit.

In ENG, a gravitational system in mechanical equilibrium has a velocity dispersion

$\sigma=\left(G M a_{0}\right)^{1 / 4} g(x)$ according to Buckingham's theorem of dimensional analysis. A particular class of astrophysical objects is characterised by a mass $M$ and length $r$ and so, the general function $g(x)$ can be approximated by a power-law $g(x)=x^{\alpha}$, i.e.

$$
\sigma(r) \propto r^{\beta} M^{\gamma}, \quad \text { where } \quad \beta=1 / 2-2 \gamma= \begin{cases}-1 / 2 & \text { if } x \gg 1 \\ 0 & \text { if } x \ll 1 .\end{cases}
$$

with transition values between these two limits for systems with $x \approx 1$. The above equation reproduces accurately the observed scaling laws in a variety of systems [6, 4]. Namely, it converges to the Faber-Jackson relation for elliptical galaxies when $x \ll 1$. In the same limit, allowing for a proportionality between isotropic velocity dispersions in pressure 
supported systems and rotation velocities in angular momentum supported ones, Eq. (3) reproduces the baryonic Tully-Fisher relation. When $x \gg 1$, this equation is the virial equilibrium relation of Newtonian gravity $r \propto \sigma / \sqrt{G \rho}$, where $\rho$ is the characteristic mass density of the system. Consequently, the galactic Faber-Jackson relation in the MONDian regime appears as the Jeans equilibrium relation in the Newtonian one. For intermediate scales $x \sim 1$, the equilibrium for local dwarf Spheroidals is also reproduced. Finally, the Fundamental plane of elliptical galaxies, for which both indices $\beta$ and $\gamma$ are directly measured, is consistent with ENG.

The outskirts of stellar globular clusters (GCs) are systems which internal stellar dynamics and structure are well understood without requiring a dark matter component. On the other hand, recent observations hint at modified dynamics for the elements in the most external orbits. The analysis of five GCs by [7] has shown that the dispersion velocity profiles in the external regions, which experience accelerations $\lesssim a_{0}$, where $x \lesssim 1$, do not follow the expected Keplerian fall. Instead, a flattening of the dispersion velocity profile is observed and a scaling law of the kind described by (3) is obtained for these systems as shown in Figure 1a). One could argue that the deviation from the Keplerian prescription is an effect of the tidal heating by the overall galactic gravitational field. However, it is hard to believe that a tidal field varying in each case could reproduce the scaling law shown in Figure 1a.

The most intriguing observable to date concerns the orbits of wide binary systems, which are ubiquitous in our Solar vicinity and their binding energy competes with the close encounters with other stars and with the tidal force of the Galaxy. Recently [8] have simulated the evolution of $5 \times 10^{4}$ binaries with $1 M_{\odot}$ in the solar neighbourhood under Newtonian gravity. The simulation takes into account the cumulative effects of the Galactic tidal field, determined observationally through the Oort constants, and encounters with field stars. The authors compute the current distribution of separations and relative velocities. The effective tidal radius for $1 M_{\odot}$ binaries is $r_{\mathrm{t}}=1.7 \mathrm{pc}$. Below this separation, the relative velocity $\Delta V$ in a binary system should scale with separation $s$ following Kepler's third law, i.e. $\Delta V \propto r^{-1 / 2}$.

In [8], the RMS relative velocity, projected along the line of sight, is plotted against the projected separation, as reproduced in Figure $1 \mathrm{~b}$ ). The above provides a test for gravity since the acceleration for particles orbiting a $1 M_{\odot}$ star falls below the transition value $a_{0}$ at separations $s=3.4 \times 10^{-2} \mathrm{pc}$. In the non-Newtonian regime of ENG, one should observe relative velocities which do not decrease with separation. Such trend follows because the binary systems are much more robust to the tidal forces than under the Newtonian regime.

The wide binary systems of the Hipparcos and the SDSS catalogues have been analysed in [9]. The sample selects stars of $\sim 0.5 M_{\odot}$, with no other close neighbours. When plotted on the projection space of Figure 1b), the distribution of stars in each catalogue lies above the prediction of [8] even after taking into account observational uncertainties. Instead, a constant relative velocity distribution is clear, supporting the non-Newtonian behaviour 

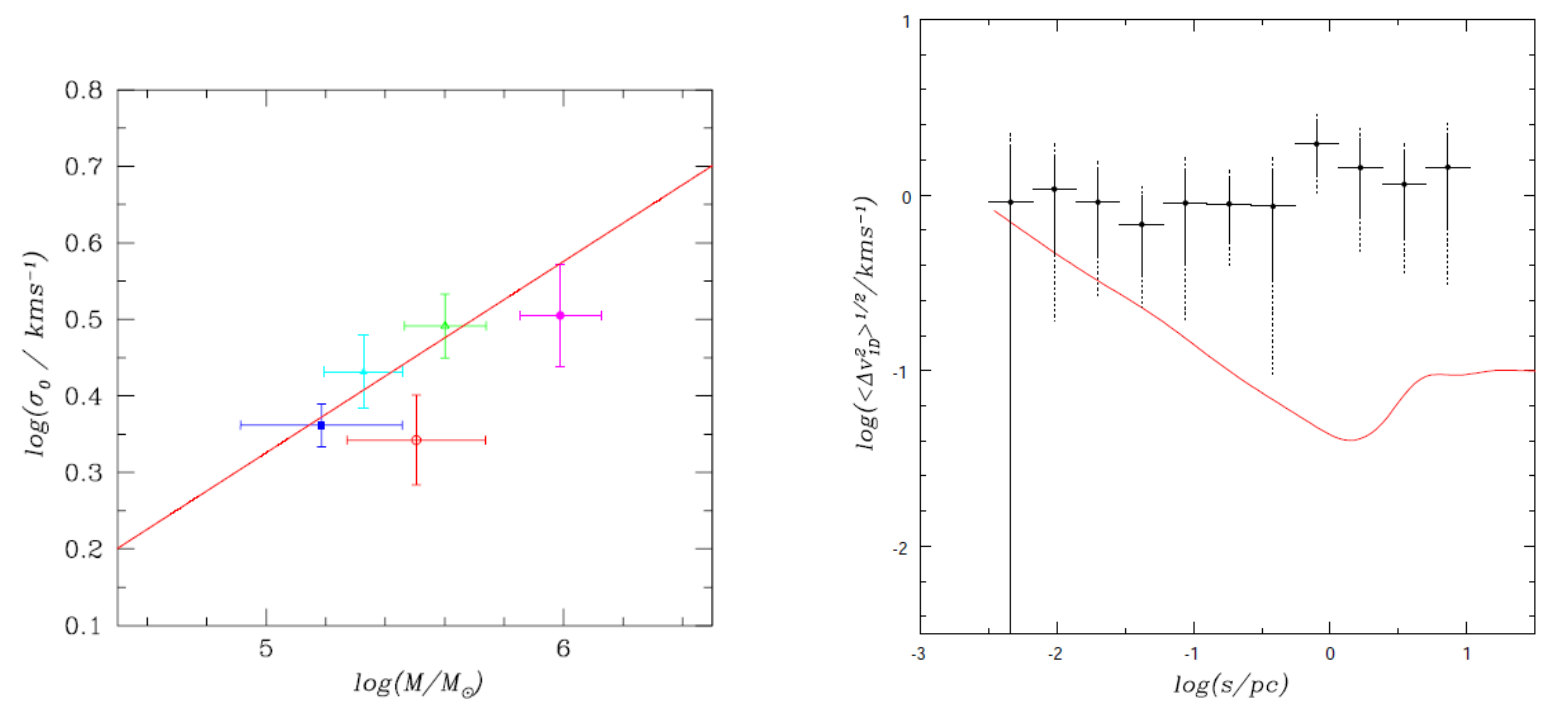

FIGURE 1. a) Scaling relation for Globular Clusters. The solid line is the best fit with a $1 / 4$ slope. b) Solid line: RMS One-dimensional, projected relative velocities as function of projected separation, from [8]. Points with error-bars: Hipparcos binary catalogue.

predicted by ENG.

In summary, the introduction of a new fundamental constant of nature $a_{0}$ yields a general scale-invariant non-relativistic theory which extends Newtonian gravity and merges naturally with MOND. ENG proves useful in explaining the dynamics of several astrophysical systems, ranging from our Solar System to galaxies. Future observations of the mentioned environments are required to test this theory in detail.

ACKNOWLEDGEMENTS: This work was supported by the following grants: UNAM-DGAPA (PAPIIT IN116210-3, IN103011-3), CONACyT: 207529, 25006, 26344. JCH acknowledges sponsorship from CTIC-UNAM and CONACYT.

\section{REFERENCES}

1. M. Milgrom, Astrophys. J. 270 (1983) 365.

2. J. D. Bekenstein, Phys. Rev. D 70 (2004) 083509 [Erratum-ibid. D 71 (2005) 069901]

3. I. Ferreras, N. E. Mavromatos, M. Sakellariadou and M. F. Yusaf, Phys. Rev. D 80 (2009) 103506

4. S. Mendoza, X. Hernandez, J. C. Hidalgo and T. Bernal, Mon. Not. Roy. Astron. Soc. 411 (2011) 226

5. Q. Exirifard, arXiv:1112.4652 [gr-qc].

6. X. Hernandez, S. Mendoza, T. Suarez and T. Bernal, Astron. Astrophys. 514 (2010) 101

7. X. Hernandez and M. A. Jimenez, arXiv:1108.4021 [astro-ph.CO].

8. Y. -F. Jiang and S. Tremaine, Mon. Not. Roy. Astron. Soc. 401 (2011) 977

9. X. Hernandez, M. A. Jimenez and C. Allen, E. P. J. C (accepted) arXiv:1105.1873 [astro-ph.GA]. 